\title{
The calculation of Hugoniots in ionic solids
}

\author{
J H Harding and A M Stoneham \\ Theoretical Physics Division. AERE Harwell. Oxon OX11 0RA. UK
}

Received 18 August 1983

\begin{abstract}
We demonstrate the quantitative prediction of Hugoniots for an ionic crystal $(\mathrm{NaCl})$ using the shell model with the best available potentials. Our calculations show that these shock wave data can be predicted quite accurately by relatively simple use of established computer codes. The Hugoniot results, together with static phase-change data, also provide a very severe test of interatomic potentials. The two sets of empirical potentials ((i) Catlow, Diller and Norgett. (ii) Sangster and Atwood) both perform much better than electron gas potentials. The same approach can be used to test potentials and to make predictions for the behaviour of solids for extreme conditions of temperature and pressure.
\end{abstract}

\section{Introduction}

Equations of state at very high temperatures and pressures have many uses. These include applications in mineralogical and geophysical studies of planetary cores, and descriptions of the behaviour of nuclear fuels in studies of hypothetical faults, the transient high-temperature behaviour needed in materials processing, and aspects of phase changes. In all these cases, knowledge of material behaviour is needed outside the regimes in which measurement is possible. The extrapolation from data for readily accessible conditions is an important area for theory, and the validation of such theory is itself of especial importance. Central to this theory are interatomic potentials, together with various prescriptions for estimating thermodynamic properties. These potentials, however, are often based on experiments or calculations corresponding to lowtemperature, low-pressure conditions. We shall demonstrate that shock wave results can be used as a stringent test of different potentials and as a check of theoretical methods for materials under extreme conditions. Our results centre on the Hugoniot data, available from shock wave experiments for many important systems.

Computer codes that calculate lattice or defect properties in ionic solids, such as the Harwell HADES code (Lidiard and Norgett 1972; for a recent survey of applications and background, see references in Catlow and Mackrodt 1982) require knowledge of the interionic potential at various distances. The HADES code uses the shell model, and assumes that the crystal potential may be written as a sum of pair potentials. The interaction between cores and shells on different ions is assumed to be purely coulombic except for the shell-shell interaction. For this shell-shell contribution there is also a short-range interaction, $\varphi_{\alpha \beta}(r)$. which is supposed to model Pauli repulsion between the ions. van der Waals terms and small covalent interactions. It is common practice to assume that the short-range interaction has a simple analytic form, such as the 
Buckingham potential:

$$
\varphi_{\alpha \beta}(r)=A_{\alpha \beta} \exp \left(-r / \rho_{\alpha \beta}\right)-C_{\alpha \beta} / r^{6}
$$

and to fit the parameters of the potential to bulk-crystal data such as elastic constants or the phonon spectrum. It has often been pointed out that this only determines the potential at the equilibrium lattice spacing whereas many calculations, especially those involving interstitials, require a knowledge of $\varphi_{\alpha \beta}(r)$ far from this distance. This has prompted many attempts to calculate short-range potentials, usually using the electron gas approximation (Catlow et al 1982, Harding and Harker 1982). However, again, the adequacy of such potentials has been tested directly only by their ability to reproduce equilibrium lattice properties.

The calculation of Hugoniot trajectories offers a way of testing the validity of the various potentials used over a range of interionic distances. It is also possible to use other data either in conjunction with or instead of Hugoniot results. In particular, the static pressure-volume data obtained with a diamond-anvil high-pressure cell are especially useful. In this paper we discuss the possibility of using such data to discriminate between various potentials available in the literature by considering the specific example of sodium chloride, using both the shock wave Hugoniot data collected by Marsh (1980) and the static pressure investigation by Bassett et al (1968) and Sato-Sorensen (1983) of the phase transition from the rock salt to the caesium chloride structure. Our discussion provides both a check of the potentials and a verification of the methods that we use in predicting properties of solids under extreme conditions.

\section{The Hugoniot equation of state}

We outline here the basic equations and assumptions we shall use, together with the methods used to obtain key quantities. Reviews of the approximations used in analysing shock wave data are given in the articles of Rice et al (1958) and Marsh (1980). Certain central results (notably the Rankine-Hugoniot relation) can be obtained from simple arguments based on one-dimensional fluid dynamics. We assume that mass and energy are conserved across the shock front and further that the shock may be considered to be an adiabatic process. This means that when we consider the possibility of a phase change in the sodium chloride system the relevant thermodynamic potential is the enthalpy, $H$.

The Rankine-Hugoniot relation states that

$$
\left(E_{1}-E_{0}\right)=\frac{1}{2}\left(P_{1}+P_{0}\right)\left(V_{0}-V_{1}\right)
$$

where $E, P$ and $V$ are the internal energy, pressure and volume (per cell, where appropriate) and the subscripts one and zero refer to the solid behind the shock front and in the undisturbed solid respectively. We shall normally take $P_{0}$ as 1 atm and $V_{0}$ as the equilibrium volume of the cell with a temperature of $300 \mathrm{~K}$. $V_{1}$ will be chosen, and values of $E_{1}$ and $P_{1}$ obtained as functions of the final temperature, which can itself be found from (2).

In calculating the pressure, we shall use the Mie-Grüneisen equation (Rice et al 1958, Decker 1965):

$$
P=-\mathrm{d} \varphi / \mathrm{d} V+\gamma(V) k T / V .
$$

Here $\varphi$ is the static part of the crystal internal energy per cell and $\gamma(V)$ the mean Grüneisen constant. It has been common to assume a particular form for the volume 
dependence of $\gamma(V)$. Examples include a Taylor expansion in which second-order and higher terms are neglected (Decker 1965), and a simple analytic form obtained from thermodynamic arguments such as that of Bassett et al (1968):

$$
\gamma=\gamma_{0}\left(V / V_{0}\right)^{A}
$$

with $\gamma_{0}$ the Grüneisen constant at volume $V_{0}$ and $A$ a fitting parameter. Such methods take no explicit account of differences in crystal structure at the phase change.

The Mie-Grüneisen equation has been compared with molecular dynamics simulations for Lennard-Jones and Morse potentials (Paskin et al 1978) and shown to give reasonable estimates of the shock-front temperature.

In our calculations we do not impose a particular analytic form on $\gamma(V)$ but obtain it by averaging the mode Grüneisen constants calculated over a mesh of points taken in the Brillouin zone. The mode Grüneisen constants are defined by

$$
\gamma_{i}=-\mathrm{d} \ln \omega_{i} / \mathrm{d} \ln V \simeq-\left(V / \omega_{i}\right) \Delta \omega_{i} / \Delta V .
$$

It is found that quite a coarse mesh (about 2000 points in the fundamental wedge of $\frac{1}{48}$ of the zone) is enough to ensure reasonable accuracy.

The accuracy of this method of calculating the mode Grüneisen constants may be checked by comparing the results with those obtained from considering the pressure derivative of the elastic constants (Daniels 1963). The results are shown in table 1 and demonstrate that this method of estimating $\gamma_{i}$ is fully acceptable.

Table 1. Calculation of mode Grüneisen constants and comparison of Daniel's results without data.

\begin{tabular}{llll}
\hline & Direction & Calculated $\gamma_{i}$ & Experimental $\gamma_{1}$ \\
\hline (100) & optic & 1.02 & \\
& & 2.81 & \\
& acoustic & 2.67 & 2.64 \\
$(110)$ & & 0.18 & 0.14 \\
& & 1.04 & \\
& & 2.81 & \\
& acoustic & 2.80 & 1.87 \\
& & 1.84 & 2.72 \\
(111) & optic & 0.87 & 0.14 \\
& & 1.04 & \\
& acoustic & 2.81 & 1.57 \\
& & 2.18 & 2.05 \\
\hline
\end{tabular}

The final relationship uses the high-temperature form of $E$, the internal energy per cell in (1):

$$
E=\varphi+E_{\mathrm{vib}}=\varphi+3 N k T
$$

with $N$ the number of ions in the unit cell.

We see now that we must calculate three important quantities: $\varphi(V)$ for equations (2) and (6) and both $\mathrm{d} \varphi(V) / \mathrm{d} V$ and $\chi(V) \equiv\left\langle\gamma_{i}(V)\right\rangle$ for (3). Given these quantities, we may calculate the temperature, pressure and other thermodynamic variables behind the 
shock. The static terms $\varphi$ and $\mathrm{d} \varphi / \mathrm{d} V$ are obtained using the Harwell PLUTO program (Catlow and Norgett 1976), and the lattice frequencies needed in (4) are found using the lattice dynamics package developed by Sangster and Rowell (1983) from the Harwell PHONONS program.

\section{Calculations of Hugoniot trajectories}

\subsection{Interatomic potentials}

In this study we shall consider three potentials: those of Catlow et al (1977; CDN), Sangster and Atwood (1978; SA) and Mackrodt and Stewart (1979; MS). The first two were obtained by fitting to bulk-crystal data, whereas the third was obtained from an electron gas calculation. All three potentials have been used in defect calculations. Further details of the potentials, their assumptions and their applications are listed in the handbook by Stoneham (1981).

\subsection{Calculation of static pressure-volume curves}

Bassett et al (1968) have investigated the rocksalt-caesium-chloride transition in sodium chloride using a diamond-anvil high-pressure cell. They find a transition pressure of about $30 \mathrm{GPa}$ and a transition volume of $-1.0 \pm 0.05 \mathrm{~cm}^{3} \mathrm{~mol}^{-1}(-3.5 \%)$. More recent experiments (Sato-Sorensen 1983) confirm this value of the transition pressure, but give a larger transition volume $(-5.8 \%)$. In this experiment the relevant thermodynamic potential is the free energy, $G$.

The vibrational contribution to the free energy is given by the expression

$$
G_{\text {vib }}=-k T \ln Q_{\text {vib }}
$$

where $Q_{\text {vib }}$ is the vibrational partition function. We have estimated this term by taking points at random in the Brillouin zone and constructing an average over them, in a similar manner to that used in calculating the Grüneisen constant. At $300 \mathrm{~K}$ the contribution of this term is about $0.06 \mathrm{eV}$, varying only slowly with lattice volume. We therefore ignore it, assuming that the free energy is given by the expression

$$
G=\varphi+P V .
$$

We have calculated the variation of this for the three potentials and find that the electron gas potential completely fails to predict the transition.

The CDN potential predicts a transition at $30 \mathrm{GPa}$ with a transition volume of $-1.2 \mathrm{~cm}^{3} \mathrm{~mol}^{-1}$, while the SA potential also predicts a transition at $30 \mathrm{GPa}$, but with a transition volume of $-1.1 \mathrm{~cm}^{3} \mathrm{~mol}^{-1}$. Considering the approximations involved, both results must be considered very reasonable. The calculations are less satisfactory, if we consider the lattice parameters at which the change takes place. These results are shown in table 2 . Here the errors in the calculation are about $5 \%$, the $\mathrm{CDN}$ potential giving the closer agreement. There are no signs of further phase changes in the predictions. This is in agreement with experiment for $\mathrm{NaCl}$, although further changes have been noted for $\mathrm{NaBr}$ and $\mathrm{NaI}$ (Yagi et al 1983).

\subsection{Shock-induced phase changes of $\mathrm{NaCl}$}

We calculate the Hugoniot trajectories for both the rocksalt and caesium chloride 


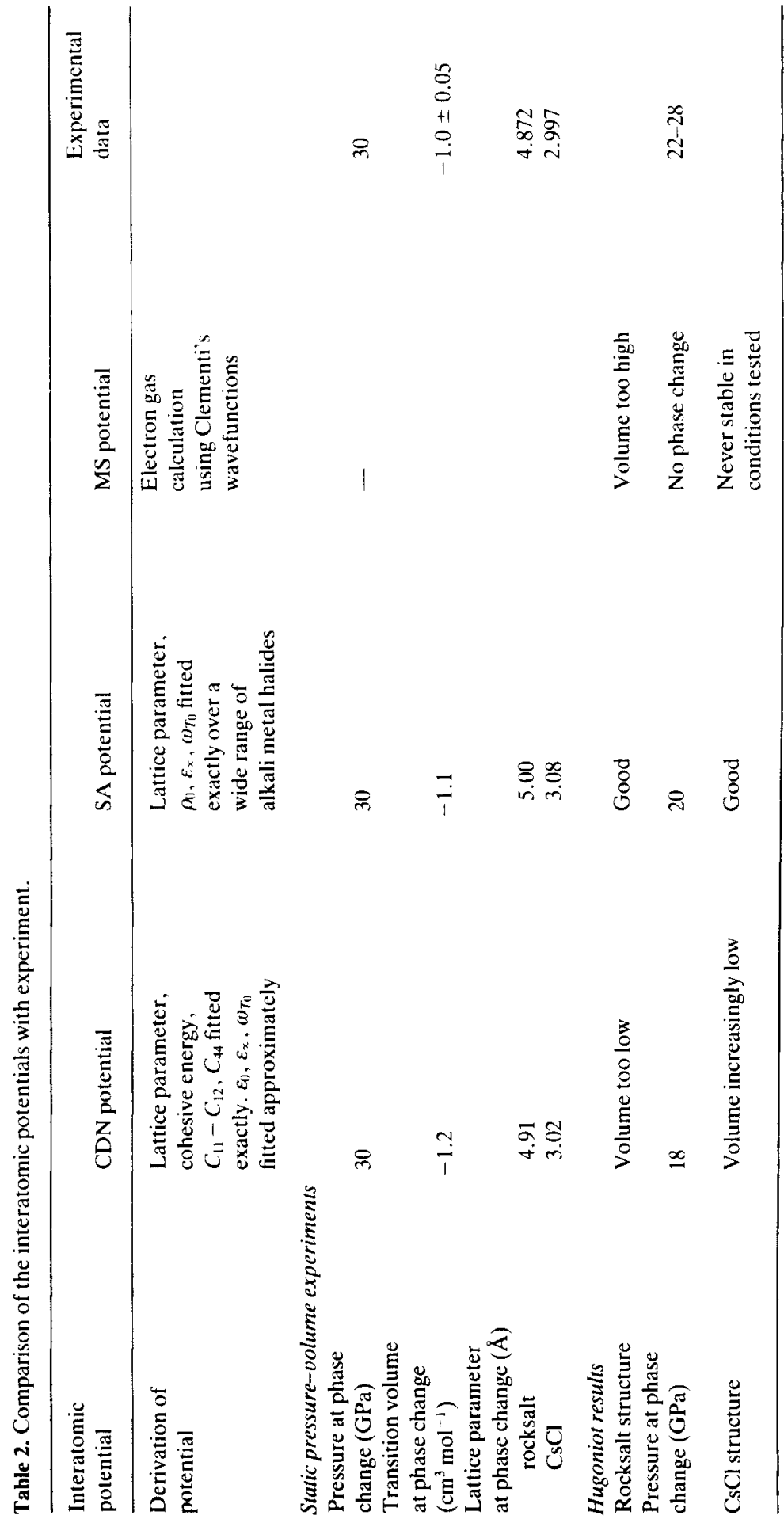


structures. These are shown in figures $1-3$ for the three candidate potentials. Calculations of the enthalpies for the two structures give an estimate for the position of the rocksalt-caesium-chloride phase transition. For the $\mathrm{CDN}$ potential this occurs at about $18 \mathrm{GPa}$. For the SA potential the pressure is about $20 \mathrm{GPa}$. The result is not precise because the slopes of the enthalpy curves at this point are very similar. The electron gas potential again fails to predict a transition at all.

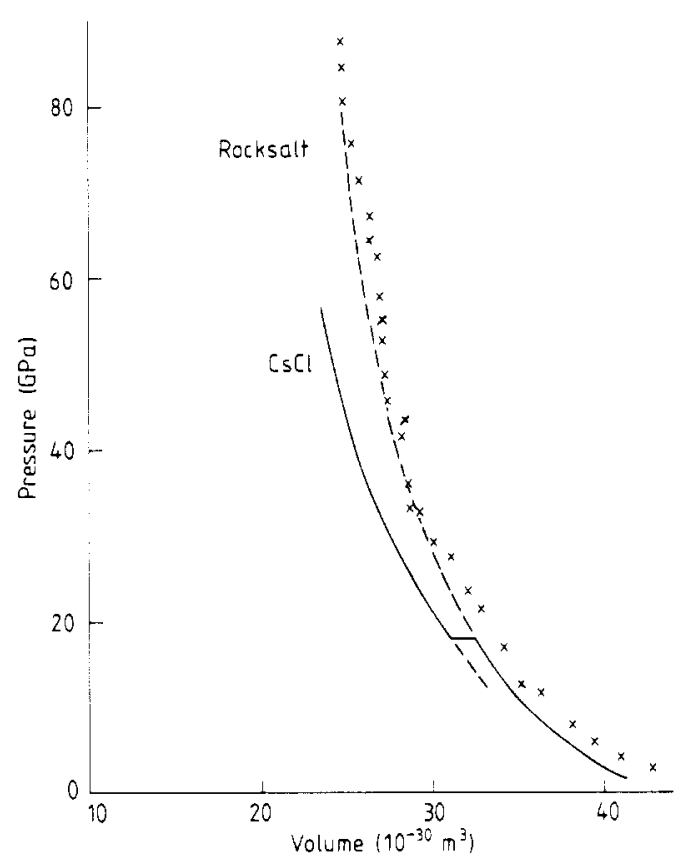

Figure 1. Hugoniot trajectory, for the $\mathrm{CDN}$ potential: full curve, stable phase ; broken curve. unstable phase; crosses, experimental.

Evidence for a phase transition in dynamic shock experiments has been given by Christian (1957), Alder (1963) and Kuzubov (1965) in the pressure range 22-27 GPa. The data from Marsh (1980) also show a pronounced change of slope at about $28 \mathrm{GPa}$.

Of the three potentials, it is clear that the SA one gives the best representation of the curve as a whole. However, it should be pointed out that in defect calculations the electron gas potentials are normally used with adjusted lattice parameters to ensure that they produce an equilibrium lattice. This adjustment has not been considered in figure 3 , and its inclusion would have the effect of shifting the calculated curve to the left. Such a change does not improve the static results, although the use of electron gas potentials might well be a more accurate procedure (with special precautions) than figure 3 would suggest.

All three potentials give a fall in the calculated mean Grüneisen constant. This is shown in figure 4 for the Sangster and Atwood potential. This agrees qualitatively with the empirical potential of equation (4). Examination of the individual mode constants (see table 1) shows that they are highly anisotropic and that the optic constants are very different from the acoustic ones. As the volume falls the optic-mode $\gamma_{i}$ tend to rise, but the acoustic-mode ones, especially those near the (100) and (110) directions, fall. The behaviour of these modes dominates the average. 


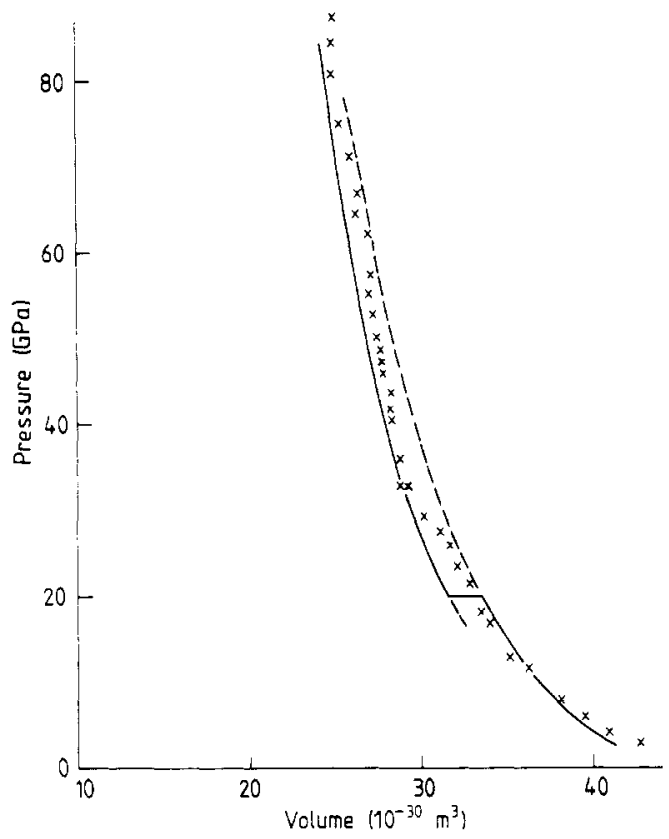

Figure 2. As figure 1, but for the SA potential.

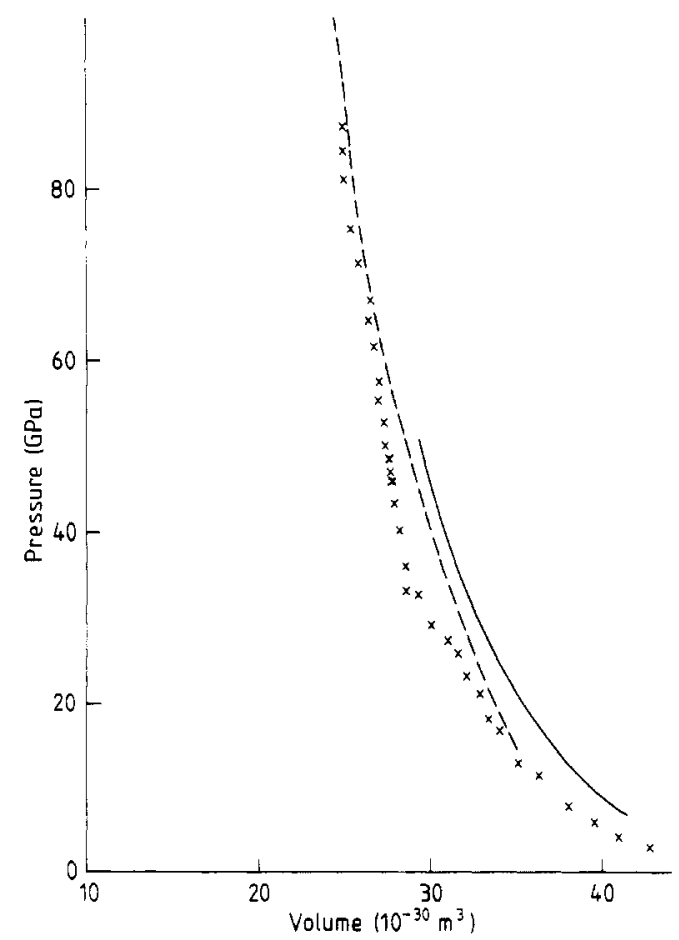

Figure 3. As figure 1, but for the MS potential. 


\subsection{Shock-front temperatures}

As outlined in $\S 2$, we may calculate the temperature and pressure corresponding to a given volume change. In the limit of high final temperature, and taking $P_{0}=T_{0}=0$, one finds simply

$$
T_{1}=\frac{\left(\varphi_{0}-\varphi_{1}\right)+\frac{1}{2}(\mathrm{~d} \varphi / \mathrm{d} V)\left(V_{1}-V_{0}\right)}{k\left[3 N+\frac{1}{2} \gamma_{1}\left(V_{0} / V_{1}-1\right)\right]}
$$

with subscripts 0,1 referring to parameters evaluated for the initial and final states. The pressure $P_{1}$ follows directly from (3) and (9). In our actual calculations we took $P_{0}=$ $1 \mathrm{~atm}$ and $T_{0}=290 \mathrm{~K}$, for which more complex expressions result. Figures 5 and 6 show the calculated shock-front temperatures from the Mie-Grüneisen equation. We first note that the rapid temperature rise justifies the use of the high-temperature approximation. The contribution of the corrections for finite temperature are important only at low pressures for which the overall contribution of the temperature-dependent effect is very small.

Shock temperatures have been measured by Kormer et al $(1965,1968)$ and by Kondo and Ahrens (1983). The measurements give widely varying values for the brightness temperatures and emissivities. The calculated shock brightness temperatures are sig-

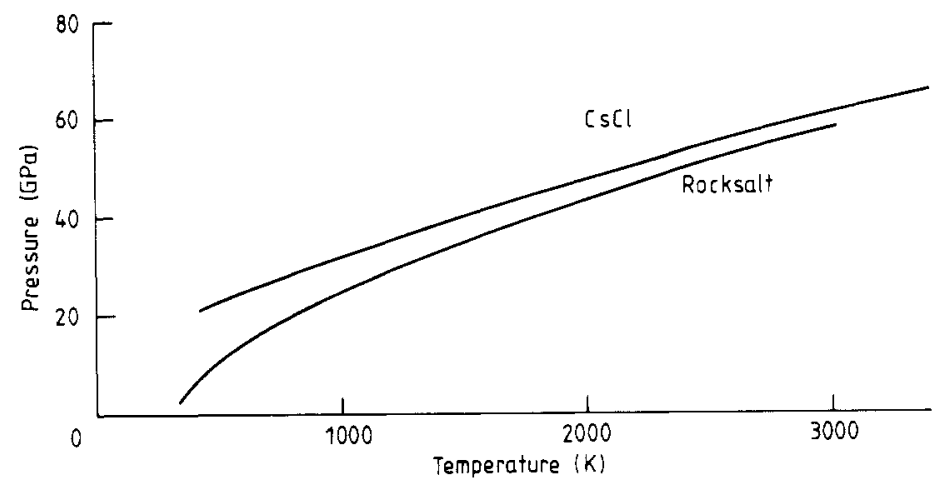

Figure 4. Plot of shock temperature against pressure, for the SA potential.

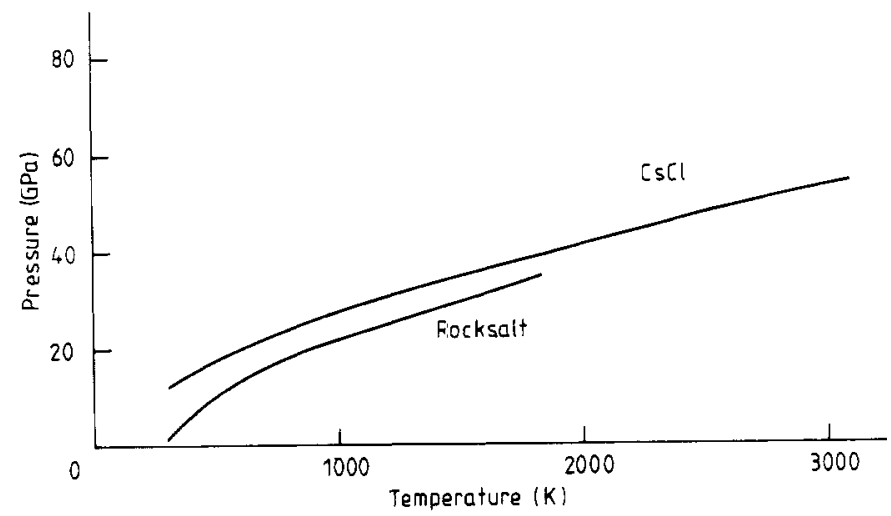

Figure 5. As figure 4, but for the CDN potential. 


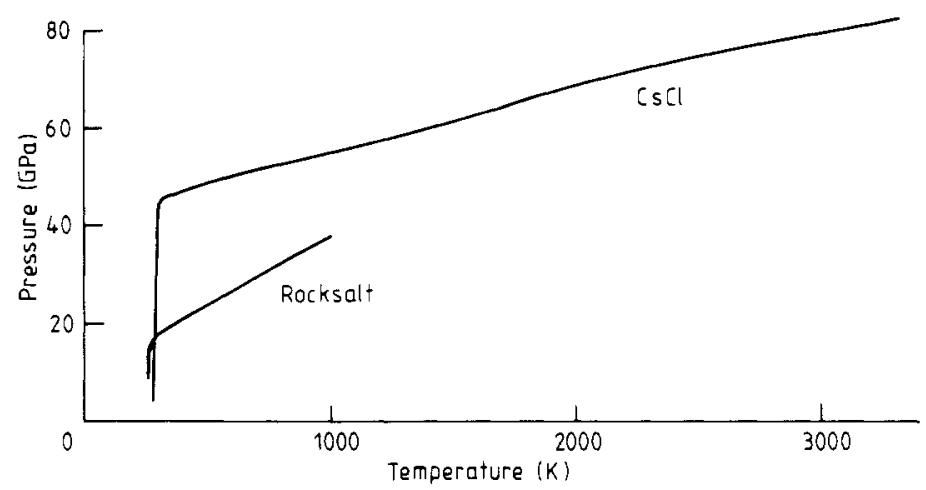

Figure 6. As figure 4, but for the MS potential.

nificantly lower than those of Kormer et al (1968) or Kondo and Ahrens (1983). Kormer et al (1968) have noted such an effect previously, and suggest that electron luminescence is responsible for the discrepancy.

The high temperatures therefore raise other questions, notably those of electronic structure changes or of defect production. Ruoff (1980) has discussed the effects of defect production, and argues that the defect pressure and the thermal pressure at a given volume change and temperature may be comparable. In particular, he suggests that the pressure may be increased by typically $20 \%$ by the presence of shock-generated vacancy defects. Gatilov and Kukshova (1981) note a massive increase in conductivity of CsI behind a shock front, which they interpret in terms of a change in character with pressure, as the ionic insulator transforms into an intrinsic-defect semiconductor, a liquid semiconductor, and finally a metallic liquid. Systems in which cations can change valence without great energy cost, so that electronic excitation is relatively easy (e.g. $\mathrm{UO}_{2}, \mathrm{FeO}$ ), should show distinctive features as they become conducting. It is just such systems that exist with substantial non-stoichiometry $\left(\mathrm{UO}_{2} \rightarrow \mathrm{UO}_{2+x} ; \mathrm{FeO} \rightarrow \mathrm{Fe}_{1-x} \mathrm{O}\right)$, and this aspect will affect any defect pressure. Changes in magnetic and spin structure are less important, but have been noted in static experiments (Yagi and Akimoto 1982) and in Hugoniots (Ogata et al 1983) for $\alpha-\mathrm{Fe}_{2} \mathrm{O}_{3}$.

\section{Conclusions}

We have calculated Hugoniot trajectories, shock-front temperatures, pressure-volume curves and phase transitions, starting from the best available interatomic potentials, and exploiting a generalised form of the Mie-Grüneisen equation. No semi-empirical value of the Grüneisen parameter is assumed. The broad consistency of both the static and the shock wave predictions with experiment verifies that our calculational methods are adequate. The precise degree of success offers a very sensitive test of the interatomic potentials, and one that is especially appropriate in extrapolating to other high-temperature, high-pressure situations. The three potentials have varying degrees of success. These are summarised in table 2 , and show that the empirical potentials have notable success even at volumes far from those for which they were fitted. It remains to be tested whether this success is typical or not, and also whether the changes resulting from defect generation and electronic excitation can be modelled equally well. 


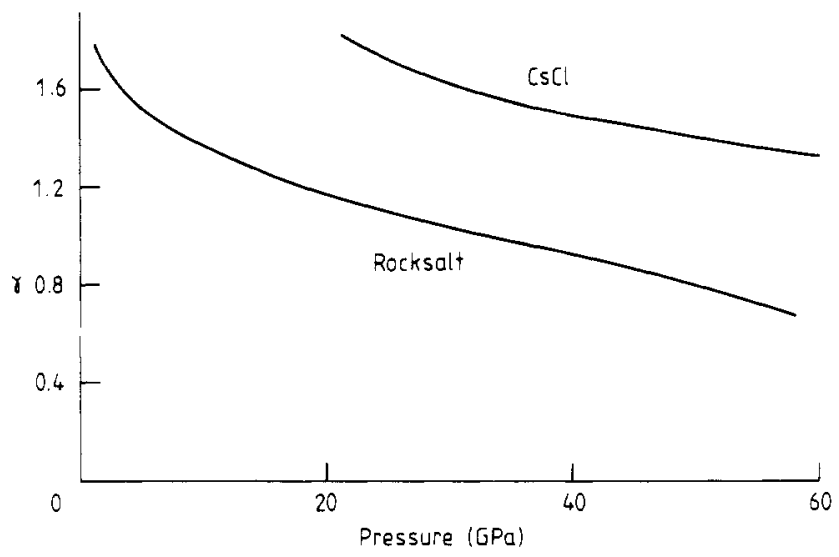

Figure 7. Plot of mean Grüneisen constant against pressure, for the SA potential.

It is also worth noting that a reasonable approximation is to ignore the volume dependence of the Grüneisen constant entirely. The second term in the Mie-Grüneisen equation only contributes about $1 \%$ to the calculated pressure and, as can be seen from the denominator of (9), the term involving $\gamma$ is always much less than the other term. Thus reasonable estimates of the behaviour of minerals can be made even without a lattice dynamical calculation. This is of some importance since the unit cells of many minerals are large and the calculation of $\gamma$ thus would be expensive.

\section{Acknowledgement}

The authors are grateful to $\mathrm{Mr} \mathrm{R}$ Ball for several useful conversations in the earlier stages of this work.

\section{References}

Alder B J 1963 Solids under Pressure ed. W. Paul and D M Warschauer (New York: McGraw-Hill) pp 478. 385

Bassett W A. Takahashi T, Mao H K and Weaver J S 1968 J. Appl. Phys. 39319

Catlow C R A, Diller K M, Norgett M J 1977 J. Phys. C: Solid State Phys. 12451

Catlow C R A, Dixon M and Mackrodt W C 1982 Computer Simulation of Solids, Springer Notes in Physics vol 166 ed. C R A Catlow and W C Mackrodt (Berlin: Springer) p 130

Catlow C R A and Mackrodt W C (eds) 1982 Computer Simulation of Solids, Springer Notes in Physics vol 166 (Berlin: Springer)

Catlow C R A and Norgett M J 1976 AERE Report No AERE-M.2936

Christian R H 1957 PhD Thesis University of California p 89

Daniels W B 1963 Lattice Dynamics ed. R F Wallis (Oxford: Pergamon) p 173

Decker D L 1965 J. Appl. Phys. 36157

Gatilov L A and Kuleshova L V 1981 Sov. Phys.--Solid State 231663

Harding J H and Harker A H 1982 AERE Rep. R10425

Kondo K I and Ahrens T J 1983 Phys. Chem. Miner. 9173

Kormer S B. Sinitsyn M V and Kirillov G A 1968 JETP Lett. 7290

Kormer S B. Sinitsyn M V. Kirillov G A and Urlin VD 1965 Sov. Phys.-JETP 21698

Kuzubov A 1965 PhD Thesis University of California $\mathrm{p} 61$ 
Lidiard A B and Norgett M J 1972 Computational Solid State Physics ed. F Herman, N W Dalton and R G Koehler (New York: Plenum) p 385

Mackrodt W C and Stewart R F 1979 J. Phys. C: Solid State Phys. 12431

Marsh S P $\left(\mathrm{ed}_{1}\right) 1980$ LASL Shock Hugoniot Data (Berkeley, California: University of California Press)

Ogata F, Kambara T, Sasaki N and Gondaira K I 1983 J. Phys. C: Solid State Phys. 161391

Paskin A. Gohar A and Dienes G J 1978 J. Phys. Chem. Solids 391307

Rice M H. McQueen R G and Walsh J M 1958 Solid State Phys. 61 (New York: Academic Press)

Ruoff A L 1980 J. Appl. Phys. $\mathbf{5 1} 6221$

Sangster M J L and Atwood R M 1978 J. Phys. C: Solid State Phys. 111541

Sangster M J L and Rowell D 1983 unpublished

Sato-Sorensen Y 1983 J. Geophys. Res. 883543

Stoneham A M 1981 AERE Report No. AERE 9598

Yagi T and Akimoto S-I 1982 Adv. Earth Plan. Sci. 1281 (High Pressure Research in Geophysics ed. S Akimoto and M H Manghani (Centre for Academic Publication of Japan/Reidel: Tokyo/Dordrecht)

Yagi T, Suzuki T and Akimoto S-I 1983 J. Phys. Chem. Solids 44135 